\title{
Application of Biowaiver Methodology for a Post-Marketing Study of Generic and Brand Name Metronidazole Tablets
}

\author{
Dhanusha Thambavita $^{1 *}$, Sampatha Fernando ${ }^{1,2}$, Privadarshani Galappatthy ${ }^{1}$, \\ and Raveendra Jayakody ${ }^{1}$ \\ ${ }^{1}$ Department of Pharmacology and Pharmacy, Faculty of Medicine, University of Colombo, Sri Lanka \\ ${ }^{2}$ Department of Chemistry, Faculty of Science, University of Colombo, Sri Lanka
}

e-mail: tmdhanusha@yahoo.com

\section{ABSTRACT}

An important component of evaluation of generic medicines is assessing their therapeutic equivalence, which is done by a comparative study with an appropriate comparator, such as an in vivo bioequivalence study or an in vitro dissolution study. In vivo bioequivalence studies are cumbersome and expensive. Their need might be waived using in vitro dissolution for selected drugs. In this post marketing study, we conducted in vitro dissolution testing according to the WHO guideline for biowaiver studies comparing a generic metronidazole product available in Sri Lanka with an appropriate comparator. The results showed that both products complied with the pharmacopoeial specifications, and that the generic (test) product is similar to the comparator product, with very rapid dissolution properties. In vitro dissolution of the test product was similar to the comparator in media with $\mathrm{pH} 1.2,4.5$, and 6.8, representing gastric, duodenal, and jejunal conditions. Based on the study findings, the tested metronidazole product demonstrates in vitro equivalence to the comparator product. Similarity of the dissolution profile indicates high probability of in vivo bioequivalence of the generic product compared to the brand name product, in the absence of bioequivalence data. According to the International Pharmaceutical Federation (FIP) biowaiver monograph for metronidazole, the quality and quantity of the excipient also needs evaluation for the two products to be considered therapeutically equivalent. Therefore, if the excipient composition is available, the decision of interchangeability of the two products could be made.

KEYWORDS: metronidazole, biowaiver, dissolution profiles, BCS

\section{INTRODUCTION}

T world, the drug regulatory authorities (DRAs) use different methods when evaluating generic products. During such evaluation, therapeutic equivalence is assessed by in vivo bioequivalence or in vitro comparative studies. Bioequivalence studies are waived according to the drug class based on the Biopharmaceutics Classification System (BCS). This process is supplemented by the international biowaiver guidelines and biowaiver monographs published by the International Pharmaceutical Federation (FIP) (1-3).According to the biowaiver guideline published by the World Health Organization (WHO), BCS Class I and Class III drugs may be considered eligible for waiver based on in vitro dissolution studies to establish therapeutic equivalence (1).
Until recently, the Sri Lankan DRA has not requested bioequivalence data at the time of registration of generic medicines, except for medicines with a narrow therapeutic index. Also, the concept of a biowaiver has not been applied in Sri Lanka. This situation may be true for other DRAs in low and middle-income countries as well. Hence, a large number of generic products are available in these countries, but a proper assessment of therapeutic equivalence has not been performed. With this background, we conducted an in vitro comparative dissolution study to determine the therapeutic equivalence of a selected product. Metronidazole was selected for our study as it is a BCS Class I drug, and an FIP biowaiver monograph is available to outline the requirements for a biowaiver (3). 


\section{MATERIALS AND METHODS}

The study consisted of three parts. First, we conducted a market survey to select a suitable generic metronidazole product for testing. Then, we performed pharmaceutical quality testing of the selected product, followed by an in vitro comparative dissolution study to determine in vitro equivalence of the product compared to the brand name product (4).

\section{Market Survey}

The market study was conducted according to the WHO operational package for assessing, monitoring, and evaluating country pharmaceutical situations (5). Twenty pharmacies were selected in and around the National Hospital of Sri Lanka from the Colombo district. A list of metronidazole products available was obtained from these selected pharmacies. The WHO guideline on selecting a comparator pharmaceutical product for bioequivalence/ biowaiver studies was used to identify the comparator metronidazole product for study $(2,6)$. Data on the solid oral metronidazole products used in the government sector were obtained from the Ministry of Health in Sri Lanka.

\section{Reagents and Materials}

The brand name pharmaceutical product of metronidazole, Flagyl 400-mg tablets (Sanofi Bangladesh Ltd., Bangladesh), purchased from the local market, was used as the comparator product. Metrogyl 400-mg tablets (Unique Pharmaceutical Labs, India), purchased from the local market, was used as the test product. The batch numbers of the products are available with the investigators. The quality testing was done according to pharmacopoeial requirements using standard reagents.

\section{Instrumentation and Equipment}

Dissolution testing was conducted in a dissolution apparatus (PTWS610, PharmaTest, Apparatebau, Germany) with an autosampler system. Absorbance of the dissolution samples was measured using a spectrophotometer (Cary50 UV-Vis, Varian, Inc., USA).

\section{Pharmaceutical Quality Testing}

Quality tests were conducted for all selected products before proceeding to biowaiver studies to confirm compliance with pharmacopoeial quality standards of the selected products. Tests were conducted according to the British Pharmacopeia (BP 2012), and the dissolution tests were conducted according to the United States Pharmacopeia (USP 2012) (7, 8). Two out of three tests were conducted according to BP 2012 for identification of metronidazole (7). Disintegration testing was conducted as a routine pharmacopoeial test before conducting the pharmacopoeial dissolution test, as an indicator of dissolution of the product.

\section{Analytical Method Validation and Quantification}

Metronidazole in dissolution samples was assayed using a validated spectrophotometric method with UV absorbance at $278 \mathrm{~nm}$. The analytical method was validated for its suitability in dissolution media with $\mathrm{pH} 1.2$, 4.5, and 6.8, according to USP 2011 (8). The linear range for method validation was selected based on the expected lowest (3\%) and highest (120\%) released concentrations of metronidazole in the $900-\mathrm{mL}$ dissolution vessel. Accuracy, linearity, and precision of the method were determined as percentage recovery, correlation coefficient $(r)$, and coefficient of variation (CV), respectively. Percent recovery falling within $\pm 15 \%$ of the true value at higher concentrations and within $\pm 20 \%$ of the true value at lower concentrations was considered as the expected accuracy of the analytical method. A correlation coefficient greater than 0.99 was used as the criterion for linearity, and CV less than $2 \%$ was used as the criterion for precision of the method.

\section{In Vitro Equivalence Study}

The in vitro equivalence study was conducted according to the biowaiver guideline published by WHO in 2006 (4). The reference product was identified based on the criteria of most available metronidazole products in the market. Dissolution media of pH 1.2, 4.5, and 6.8 were prepared according to the standard buffer solutions given in USP 2012 and deaerated prior to dissolution testing. A validated media degassing method was used for the deaeration of the dissolution media. In vitro dissolution testing was performed for 12 dosage units of both the test product and the comparator product in $900 \mathrm{~mL}$ of standard buffer media in $\mathrm{pH} 1.2,4.5$, and 6.8 at $37^{\circ} \mathrm{C} \pm 0.5^{\circ} \mathrm{C}$. The paddle method at $75 \mathrm{rpm}$ was used for the dissolution of tablets. Samples were withdrawn at 10, 15, 20, 30, 45, and 60 minutes, and total dissolution time was 60 minutes. Absorbance was measured at $278 \mathrm{~nm}$. Percent dissolution of the drug was calculated at each time point for the 12 dosage units of both products using a calibration curve. Cumulative dissolution profiles were generated for each time point.

In biowaiver studies, in vitro dissolution of the generic product was compared with that of a comparator product under standardized conditions. The dissolution profiles were compared using a similarity factor $\left(f_{2}\right)(2)$. 


\section{RESULTS AND DISCUSSION}

\section{Market Study}

Out of 32 registered solid oral metronidazole products in the database of the Sri Lankan DRA, only 10 products were available in the surveyed area in Colombo (9). Each pharmacy studied had two or three brands at any given time. Of the 20 pharmacies studied, the selected test product was available in 17 pharmacies and the selected comparator product was available in 16 pharmacies (Table 1). According to the data obtained from the Ministry of Health, the selected test product was used in government hospitals during the study period. Therefore, the selected test product was the most widely available solid oral metronidazole product in both the private and government sectors in Sri Lanka. The brand name product of metronidazole (Flagyl) was used as the comparator, and this happened to be the second most widely used brand of metronidazole.

\section{Quality Testing}

Results of quality testing of the test product and comparator product are summarized in Table 1. The pharmacopoeial dissolution test results represented in Figure 1. These results indicate that both products satisfied the pharmacopoeial quality requirements.

Table 1. Results of Quality Testing of Two Metronidazole Products

\begin{tabular}{|c|c|c|}
\hline Test (BP 2012) & Test Product & Comparator Product \\
\hline Identification & & \\
\hline Test A & Complied & Complied \\
\hline Test B & Complied & Complied \\
\hline Assay (95-105\%) & 98.01 & 98.44 \\
\hline $\begin{array}{l}\text { Uniformity of Weight } \\
\qquad( \pm 5 \%)\end{array}$ & $\pm 0.02354 \mathrm{mg}$ & $\pm 0.03096 \mathrm{mg}$ \\
\hline $\begin{array}{l}\text { Disintegration Time } \\
\text { (min) }\end{array}$ & 2.17 & 4.24 \\
\hline $\begin{array}{l}\text { Resistance to Crushing } \\
\left(\mathrm{kg} / \mathrm{cm}^{2}\right) \text {, Mean (Range) }\end{array}$ & $215.3(177-254)$ & $182.5(159-198)$ \\
\hline Dissolution (USP 2012)* & $\begin{array}{l}>85 \% \text { dissolved } \\
\text { within } 10 \text { minutes }\end{array}$ & $\begin{array}{c}>85 \% \text { dissolved } \\
\text { within } 10 \text { minutes }\end{array}$ \\
\hline
\end{tabular}

*Not less than $85 \%$ of the labeled amount of metronidazole should dissolve within 60 minutes.

\section{Analytical Method Validation of Metronidazole in Dissolution Samples at Different pH Levels}

The correlation coefficient $(r)$ between concentrations and responses (absorbance) in dissolution media with $\mathrm{pH} \mathrm{1.2,}$ 4.5 , and 6.8 were $0.998,0.996$, and 0.995 , respectively. Spiked samples were used for the determination of accuracy. Average recovery percentages of the spiked

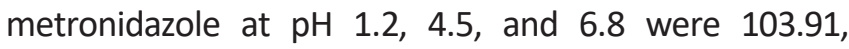
100.33 , and 97.48 , respectively. Precision of the analytical method was calculated as the CV of nine determinants for the three $\mathrm{pH}$ levels $(1.2,4.5$, and 6.8$)$ separately. The $\mathrm{CV}$ of the responses of the nine determinants was less than $15 \%(0.453,0.904$, and 0.67 for $\mathrm{pH} 1.2,4.5$, and 6.8 , respectively).

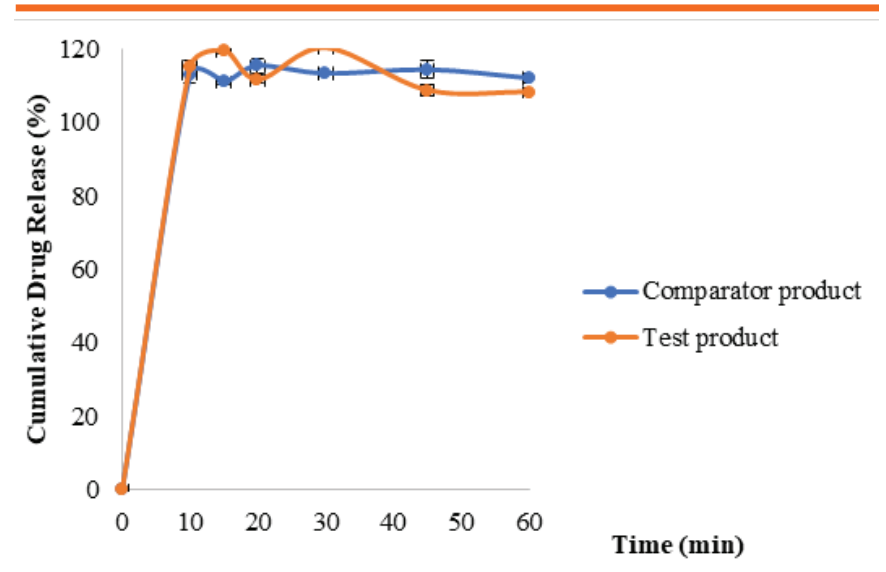

Figure 1. Dissolution profiles of the two metronidazole products in $0.1 \mathrm{~N}$ hydrochloric acid).

\section{In Vitro Dissolution Testing}

The cumulative dissolution profiles of the test product and reference product in dissolution buffers at $\mathrm{pH} 1.2,4.5$, and 6.8 are presented in Figures 2, 3, and 4, respectively. According to the average drug release percentage in the Table 2, the test product and comparator product showed more than $85 \%$ dissolution in less than 15 minutes at all three $\mathrm{pH}$ levels. Therefore, both products can be categorized as very rapidly dissolving dosage forms. If both products are very rapidly dissolving, further dissolution profile comparison using $f_{2}$ calculation is not necessary for similarity assessment (4). This result is consistent with a previous study done by Reddy et al., in which the same test product and the same comparator product showed very rapid dissolution (10). However, according to Löbenberg et al., metronidazole tablets manufactured by Sanofi-Aventis (Flagyl) required $60 \mathrm{~min}$ to release more than $85 \%$ of its dose (11). Even though there are similarities among the products studied by us and by Reddy et al and Löbenberg et al., the differences in the percentage of drug release findings by Löbenberg et al, may be due to several variables, such as the characteristics of the active pharmaceutical ingredient or the quality and composition of the excipient $(10,11)$. 
Table 2. Mean Cumulative Drug Release of Two Metronidazole Products in Different $\mathrm{pH}$ Media.

\begin{tabular}{|c|c|c|}
\hline $\begin{array}{c}\text { Dissolution } \\
\text { Medium pH }\end{array}$ & $\begin{array}{c}\text { Drug Release of Test } \\
\text { Product (\%) }\end{array}$ & $\begin{array}{c}\text { Drug release of } \\
\text { Comparator } \\
\text { Product (\%) }\end{array}$ \\
\hline $\mathbf{1 . 2}$ & 100.62 & 102.23 \\
\hline $\mathbf{4 . 5}$ & 94.25 & 100.14 \\
\hline $\mathbf{6 . 8}$ & 95.32 & 97.69 \\
\hline
\end{tabular}

The disintegration time was 5 minutes for both these products. This fast disintegration aids rapid dissolution of the products. According to the biowaiver monograph, metronidazole is a highly soluble drug $(64.8 \mathrm{mg} / \mathrm{mL}$ at room temperature, $\mathrm{pH} 1.2$ ) and has a dose-solubility ratio less than 250 for a $500-\mathrm{mg}$ dose in the $\mathrm{pH}$ range 1.0-7.0 at $37{ }^{\circ} \mathrm{C}$. Thus, our study finding is in agreement with the published literature on the dose-solubility ratio of metronidazole.

According to the biowaiver monograph of metronidazole, in addition to the in vitro dissolution similarity, the test product should contain only the excipient given in the monograph in usual amounts (3). The monograph has specifically mentioned that if the test product contains sorbitol, sodium lauryl sulfate, or propylene glycol, then the test product needs to be qualitatively and quantitatively identical to its comparator with respect to these excipients. According to the excipient information given in the biowaiver monograph, the test product does not contain these three excipients. Because the test product and comparator product were both mentioned in the excipient table in the monograph, it can be assumed to have qualitatively met the excipient specifications. In our study, we did not have direct access to the pharmaceutical manufacturers to obtain necessary excipient information. Instead, we obtained the list of excipients of both products from the biowaiver monograph for metronidazole. Thus, notwithstanding the in vitro similarity showed by the tested metronidazole tablet, further investigation on specific excipient composition is required to establish the therapeutic interchangeability to the brand name product. Therefore, if a biowaiver study is considered, in addition to the similarity of dissolution profiles, the composition of the excipient must be similar. This aspect was not covered in this study, as the information on excipients was not available to us. However, this study does show that a biowaiver for metronidazole may be requested from a regulatory authority by providing in-vitro dissolution data and the excipient information for comparison. This study adds to the literature on the value of BCS-based biowaiver studies to predict the therapeutic equivalence of generic drug products to brand name drug products. In low and middle-income countries, such as Sri Lanka, where the regulatory authority does not request bioequivalence data for registration of most pharmaceuticals and considering the high cost of bioequivalence testing, this study shows that a biowaiver could be used to show therapeutic equivalence along with excipient information. This information would be useful when revising the bioequivalence criteria for registration of pharmaceuticals.

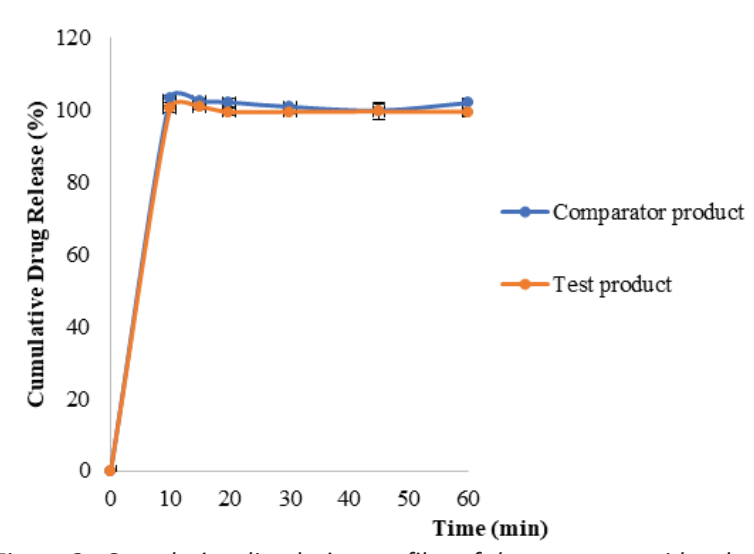

Figure 2. Cumulative dissolution profiles of the two metronidazole products in pH 1.2 media.

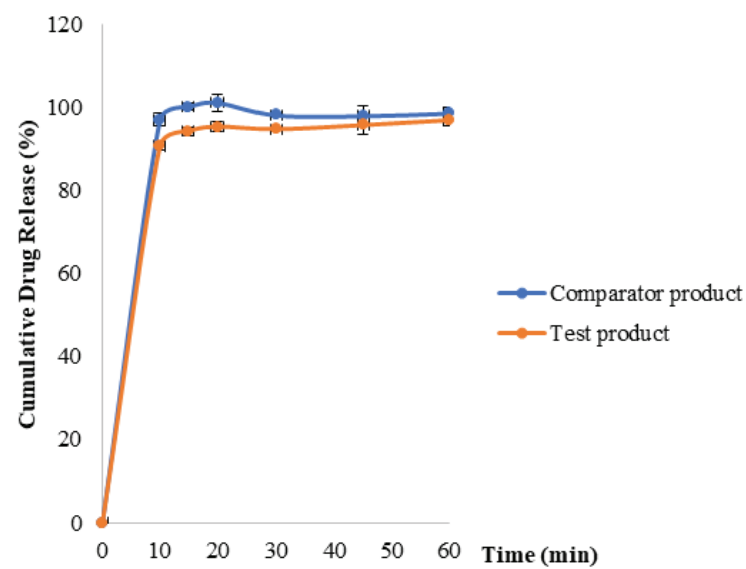

Figure 3. Cumulative dissolution profiles of the two metronidazole products in $\mathrm{pH} 4.5$ media.

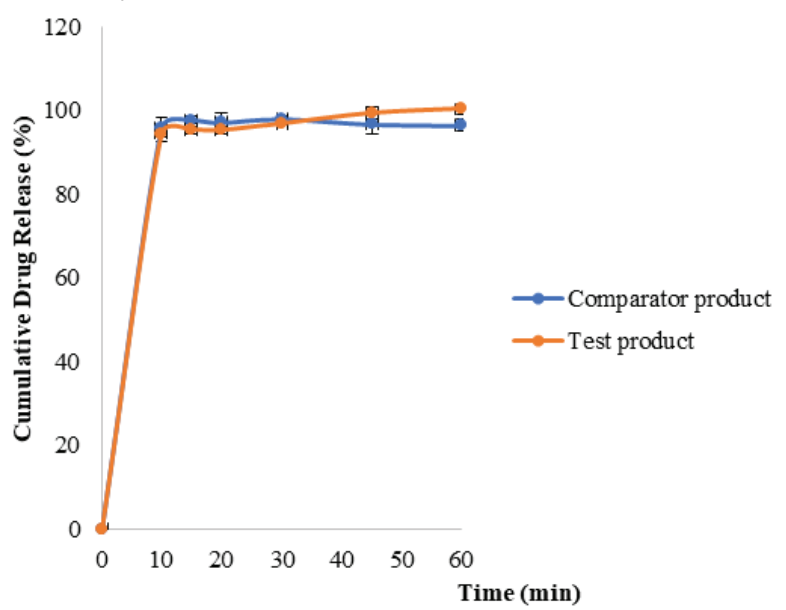

Figure 4. Cumulative dissolution profiles of the two metronidazole products in pH 6.8 media. 


\section{CONCLUSION}

The generic metronidazole product tested demonstrates in vitro equivalence to the comparator product. If information on excipient composition is available, therapeutic interchangeability of the two products could be established. Although further excipient information is needed for a biowaiver decision, similarity of the dissolution profile obtained indicates high probability of in vivo bioequivalence of the test product to the brand name product in the absence of bioequivalence data.

\section{ACKNOWLEDGEMENTS}

The study was funded by the University of Colombo collaborative research grant. The authors thank State Pharmaceutical Manufacturing Corporation (SPMC) Sri Lanka for providing metronidazole reference standard and Ms. D. M. D. Chitra and Ms. P. Nugegoda for their technical assistance. We thank the research writing support program, faculty of medicine, and AuthorAID resources at International Network for the Availability of Scientific Publications (INASP) for writing assistance.

\section{CONFLICT OF INTEREST}

The authors disclosed no conflicts of interest related to this study.

\section{REFERENCES}

1. WHO Expert Committee on Specifications for Pharmaceutical Preparations. 49th Ed.. WHOTechnical Report Series, no. 992. World Health Organization: Geneva, Switzerland,2015.

2. Approved Drug Productswith Therapeutic Equivalence Evaluations.32nd Ed.U.S. Department of Health and Human Service Food Drug Administration: Rockville, MD, 2012.

3. Rediguieri, C. F.; Porta, V.; Nunes, D. S. G.; Nunes, T. M.; Junginger, H. E.; Kopp, S.; Midha, K. K.; Shah, V. P.; Stavchansky, S.; Dressman, J. B.; Barends, D. M.Biowaiver monographs for immediate release solid oral dosage forms: metronidazole. J.Pharm.Sci. 2011,100,1618-1627.DOI: 10.1002/jps.22409.
4. Multisource (generic) pharmaceutical products: guidelines on registration requirements to establish interchangeability.In: WHO Expert Committee on Specifications for Pharmaceutical Preparations. 40th Ed. WHO Technical Report Series, No. 937. World Health Organization: Geneva, Switzerland, 2006, Annex 7.

5. WHO operational package for assessing, monitoring and evaluating country pharmaceutical situations.World Health Organization:Geneva, Switzerland, 2007. Available at http:// apps.who.int/medicinedocs/documents/s14877e/s14877e.pdf. Accessed April 1, 2018.

6. Guidance on the selection of comparator pharmaceutical products for equivalence assessment of interchangeable multisource (generic) products, WHO Technical Report Series, no. 902. World Health Organization:Geneva, Switzerland, 2002, Annex 11.

7. Metronidazole tablet. In: The British Pharmacopoeia; The British Pharmacopoeia Commission: London, 2012.

8. Metronidozole tablets. In: The United States Pharmacopoeia and National Formulary USP 35-NF 30; The United States Pharmacopoeial Convention, Inc.: Rockville, MD, 2012.

9. Cosmetics Devices and Drugs Authority. Products Directory: 2018; http://nmra.gov.lk/index.php?proType $=1 \&$ manucountry $=0 \& m$ anufacturer=0\&importer=0\&keyword=metronidazole\&btnSub mit=SEARCH\&task=search\&option=com_product\&ltemid=127, Accessed April 1, 2018.

10. Reddy, N.H.; Patnala, S.; Löbenberg, R.; Kanfer, I. In vitro dissolution of generic immediate-release solid oral dosage forms containing BCS class I drugs: comparative assessment of metronidazole, zidovudine, and amoxicillin versus relevant comparator pharmaceutical products in South Africa and India. AAPS PharmSciTech. 2014, 15, 1076-1086.DOI: 10.1208/s12249014-0135-6.

11. Löbenberg, R.; Chacra, N.B.; Stippler, E. S.; Shah, V. P.; DeStefano, A. J.; Hauck, W. W.; Williams, R. L. Toward global standards for comparator pharmaceutical products: case studies of amoxicillin, metronidazole, and zidovudine in the Americas. AAPS J. 2012, 14, 462-472.DOI: 10.1208/s12248-012-9350-9. 